\title{
Perception of concatenative vs. neural text-to-speech (TTS): Differences in intelligibility in noise and language attitudes
}

\author{
Michelle Cohn ${ }^{1}$, Georgia Zellou ${ }^{1}$ \\ ${ }^{1}$ Phonetics Laboratory, Department of Linguistics, UC Davis, USA \\ \{mdcohn, gzellou\}@ucdavis.edu
}

\begin{abstract}
In this study, we test two questions of how users perceive neural vs. concatenative text-to-speech (TTS): 1) does the TTS method influence speech intelligibility in adverse listening conditions? and 2) does a user's ratings of the voice's social attributes shape intelligibility? We used identical speaker training datasets for a set of 4 speakers (using AWS Polly TTS). In Experiment 1, listeners identified target words in semantically predictable and unpredictable sentences generated in concatenative and neural TTS at two noise levels ( $-3 \mathrm{~dB},-6 \mathrm{~dB} \mathrm{SNR})$. Correct word identification was lower for neural TTS than concatenative TTS, in the lower SNR, and for semantically unpredictable sentences. In Experiment 2, listeners rated the voices on 4 social attributes: sentences generated with neural TTS were rated as more human-like, natural, likeable, and familiar than concatenative TTS utterances. Furthermore, we observed individual variation in a given listener's SPIN accuracy measures: how humanlike/natural they rated the neural TTS voice was positively related to their speech-in-noise accuracy. Together, these findings show that the TTS method influences both intelligibility and social judgments of speech - and that these factors are linked. Overall, this work contributes to our understanding of the nexus of speech technology and human speech perception.
\end{abstract}

Index Terms: concatenative TTS, neural TTS, speech-innoise perception, language attitudes

\section{Introduction}

The recent pervasiveness of household voice-activated artificially intelligent (voice-AI) devices (e.g., Google Home, Amazon Echo) means that users are interacting with synthetic, text-to-speech (TTS) voices in their everyday lives. Yet, whether the speech generated by these modern systems is equally intelligible to listeners across different listening conditions (e.g., background talkers, music, fans, etc.) has not been thoroughly explored (cf., [1]). Further, there have been increasing efforts to make voice-AI speech as naturalistic as possible, resulting in more seamless, connected speech. For example, the application of long term short term (LSTM) neural networks in TTS (e.g., Wavenet in [2]; for a review see [3]) has resulted in more naturalistic connected speech that is rapidly being adopted industry-wide [4]. How these differences in TTS methods impact the intelligibility of the speech of voice-AI, however, is an open question.

In the present study, we consider how different TTS methods influence users' perception of synthetic speech. First, we test whether TTS generated via concatenative versus neural synthesis methods might result in differences in intelligibility during speech-in-noise perception. In concatenative TTS, individually recorded utterances are chunked into segments then re-combined via unit selection, which listeners perceive as having prosodic peculiarities (cf. [5]). Further, the concatenation process, particularly for prerecorded real words, lacks the between-word coarticulation, or articulatory overlap, that is present in natural, connected speech [6]. Autoregressive neural TTS methods, on the other hand, are conditioned on all previous utterances, as well as the immediately preceding segmental content (the local acousticphonetic context), resulting in significantly higher perceived 'naturalness' ratings by listeners [2]. This reported difference in perceived naturalness leads us to ask a second question: are there differences in how users rate social-indexical characteristics of the neural and concatenative TTS voices, and are these judgments related to intelligibility?

In the following sections, we provide a background on the speech-in-noise perception literature, reviewing differences based on 'clear' and 'connected' speech ( $\$ 1.1)$, the impact of semantic context (\$1.2), and finally individual differences in listeners' ratings of social attributes and how they impact intelligibility in the human-human literature $(\S 1.3)$.

\subsection{Intelligibility and connected speech}

Difficulty in perceiving speech in the presence of background noise has been well attested in the literature [7]-[11], particularly for adults and children with hearing impairment [8]-[10]. Competing auditory signals (e.g., a lawn mower, other talkers, etc.) can interfere with a listener's ability to hear a speaker's intended message, whether they are a dinner companion in a noisy restaurant or if they are a voice-AI system in a noisy room. While work has shown that the type of masking noise has an effect on perception (e.g., multitalker babble versus white noise in [11]), the acoustic-phonetic properties of the speech signal is also a factor. For example, in a comparison of intelligibility of natural human and synthetic speech, listeners showed the lowest comprehension for TTS voices [12]. While more connected speech, such as that generated by neural TTS, is more 'natural' sounding to human listeners, there are competing theoretical accounts as to how, and what features of, connected speech might impact intelligibility. For one, less effortful and casual speech results in shorter durations and less canonical segments than in speech produced in a clear manner ('clear speech' [13]). As a result, there is less robust content for listeners to glean from a noisy signal. Therefore, one prediction is that speech-in-noise perception for neural TTS utterances will be more difficult for listeners, relative to concatenative TTS.

On the other hand, some work has shown that speakers do not monotonically reduce all aspects of their speech when speaking casually, but rather target and maintain helpful 
contrasts for listeners, a communicatively strategic adjustment. For example, [14] found that speakers produced greater coarticulation on words with more phonological neighbors (i.e., words could be confused with similarsounding words) when their listener's intelligibility was emphasized; this had a consequence on perception: listeners displayed faster reaction times for these words produced with greater coarticulation in a lexical decision experiment, than when the words were produced with less coarticulation. One possibility is that greater coarticulatory overlap, or segmental connectedness, give listeners additional cues to 'chunk' the speech signal, attributing sets of sounds to the same word, rather than another (e.g., team-ate vs. tea-mate). Accordingly, one prediction is that more connected TTS methods (i.e., neural TTS) might improve intelligibility across increasingly noisy contexts, with greater cues for identifying words distributed across adjacent segments.

Another possibility is that speech trained on datasets of a professional voice actor's productions used in modern voiceAI systems (e.g., Siri, Alexa) may display less coarticulation and connected speech in general. There is evidence that voice actors produce clearer speech than individuals without training (cf. 'speaker's formant' in [15]). Therefore, it is possible that there will be no difference in speech-in-noise reception for concatenative and neural TTS methods, as both will show equal degrees of coarticulatory overlap given that they were both trained on identical datasets where the clarity of the original speaker is controlled.

\subsection{Speech-in-noise and semantic predictability}

In addition to acoustic-phonetic clarity (e.g., 'clear' versus 'casual' speech), listeners also use semantic context from an utterance to aid word identification in noise (e.g., [16], [17]). How listeners integrate this context, however, may differ according to how their interlocutor is speaking [18]. For example, [18] found worse keyword identification in semantically anomalous contexts, but less of a decline when the utterance was produced in clear speech compared to casual speech (both recorded by a female talker). In other words, the effect of semantic context on word intelligibility is mediated by the acoustic-phonetic properties of the utterance. Thus, we ask whether the effect of semantic predictability during speech-in-noise perception differs across concatenative and neural TTS. One prediction is that neural TTS, conditioned on the previous utterances (i.e., long-term) as well as the immediately preceding acoustic context (i.e., short-term), will improve intelligibility of the final target word since it provides more robust acoustic-phonetic cues in the signal (e.g., coarticulation) which listeners will be able to leverage when semantic context is not helpful. On the other hand, if listeners are not able to disentangle these prosodic cues from the competing background noise, then an alternative prediction is that neural TTS will result in even lower accuracy for low predictability sentences. This is in line with evidence of reduced intelligibility for more casual and connected speech, relative to clear speech [19], [20]

\subsection{Individual differences in speech-in-noise perception}

At the same time, there is a great deal of variation among listeners in speech-in-noise tasks (cf. [21], [22]). For one, a listeners' familiarity with the speech variety has shown to directly impact speech-in-noise perception [16], [17], [23]. For example, [16] found that the intelligibility benefit of semantically predictable contexts is reduced when the speaker produces a dialect that the listener is unfamiliar with. Related work has demonstrated that listeners' attitudes toward nonnative accented speech are directly linked to intelligibility [24]. Others have shown differences in intelligibility and participants' 'likeability' of certain synthetic voices, but a direct relationship between TTS voice and rating was not observed [25]. One possibility is that, in that study, there was a confound between the socio-indexical characteristics of the voice itself and how intelligible it was. Here, we disentangle these factors by holding the 'speaker' constant (e.g., AWS Polly 'Salli' voice), but manipulating the type of TTS method.

Therefore, an additional important consideration in the present study is whether individual language attitudes of the TTS voices may shape their intelligibility under difficult listening conditions. In particular, the current study tests whether there are differences in how listeners perceive neural and concatenative TTS voices for four dimensions: how 1) machine-like / human-like, 2) unfamiliar / familiar, 3) eerie / natural, and 4) how unlikeable / likeable the voice sounds. We predict that there will be a relationship between these ratings and intelligibility: in particular, that voices rated as more human-like, natural, and familiar will show intelligibility benefits, in line with the work in human-human interaction.

\subsection{Current Study}

The present study consisted of two experiments. In Experiment 1, we compare keyword identification accuracy of sentences presented in noise, comparing semantically predictable and unpredictable contexts, for speech generated from two different types of TTS methods: neural and concatenative TTS. Both TTS types were trained on identical speaker datasets ('Joanna', 'Salli', etc.). Using TTS voices generated by distinct methods allows us to explicitly test predictions about the role of neural versus concatenative speech on intelligibility. It also provides a benefit for direct replication of this study in other labs, where idiosyncratic properties of recruited speakers may otherwise contribute to differences in their relative intelligibility. In Experiment 2, we collect each participant's ratings of the four social attributes of interest: human-likeness, familiarity, naturalness, and likeability of each voice. We first test whether there are systematic differences in these ratings by TTS Condition (neural vs. concatenative) and then relate patterns of variation directly to intelligibility ratings in Experiment 1.

\section{Experiment 1: Intelligibility in Noise}

\subsection{Methods}

\subsubsection{Participants}

Participants consisted of 28 native English speakers (24 female; mean age $=19.29$ years, $\mathrm{sd}=1.41$ years) recruited through the UC Davis Psychology subjects pool. Overall, 26 participants had experience with at least one voice-AI system: 15 for Amazon Alexa, 8 for Google Assistant, and 11 for Apple's Siri.

\subsubsection{Stimuli}

We selected 192 sentences from the Speech Perception in Noise (SPIN) test [26], where monosyllabic target words occurred sentence-finally. Half of the target words were semantically predictable based on context (e.g., "The boat sailed along the coast."), while the other half were 
semantically unpredictable (e.g., "Miss Brown might consider the coast."). Using AWS Polly (US-English), all 192 sentences were generated with both concatenative TTS and neural TTS for 4 adult female Amazon Polly voices (US-English): 'Salli', 'Kendra', 'Kimberly', and 'Joanna'. All sound files were resampled to the lower sampling rate of the two (neural TTS $22,050 \mathrm{~Hz})$ and amplitude-normalized $(60 \mathrm{~dB})$. The beginning and end of each sentence was padded with $800 \mathrm{~ms}$ of silence; as a result, when mixed with the speech-shaped noise, all target sentences were gated into noise. Next, we generated speech-shaped noise using the long-term average spectrum (LTAS) for all sentences combined [27], [28]. Then, all sentences were combined with the speech-shaped noise at two signal to noise ratios: $-3 \mathrm{~dB}$ and $-6 \mathrm{~dB}$ SNR [29].

\subsubsection{Procedure}

Participants completed the experiment in a sound-attenuated booth in the UC Davis Phonetics Lab. Participants were seated in the booth facing a computer monitor and keyboard and wearing over-ear headphones (Seinheiser Pro). On each trial, participants heard a sentence and were prompted to type the last word of the sentence using the keyboard. The 192 sentences were presented equally across the 8 voices, 2 TTS conditions, and 2 SNRs; which sentence was presented in which condition was fully randomized between subjects. Finally, participants completed a short hearing screen (250$8000 \mathrm{~Hz}$ [30]). Data for participants who did not pass the screening were excluded from the analysis.

\subsection{Word identification analysis \& results}

Keyword accuracy on each trial was coded as binomial data (1 $=$ correct word identification, $0=$ incorrect) automatically using string matching. Trial accuracy ( 1 or 0$)$ was modeled with a mixed effects logistic regression with the lme4 $\mathrm{R}$ package [31]. Fixed effects included TTS Condition (2 levels: concatenative, neural), Signal to Noise Ratio (2 levels: $-3 \mathrm{~dB}$ SNR, -6 dB SNR), Semantic Predictability (2 levels: low, high), and all possible interactions. Random effects included by-Listener random intercepts and by-Listener random slopes for TTS method, SNR, and Semantic Predictability conditions. Additionally, we included by-Talker random intercepts to account for variation in baseline intelligibility for each speaker dataset. Contrasts were sum coded.

Table 1 provides the summary statistics of the accuracy model. The model revealed significant main effects and interactions. First, there was a main effect of TTS Condition. Listeners were less accurate at keyword identification for neural TTS than for concatenative TTS. SNR level and Semantic Predictability were also computed as significant main effects: listeners were less accurate at keyword identification for sentences presented at a lower SNR (-6 dB SNR), relative to a higher SNR; and listeners were less accurate at identifying words occurring in low semantic predictability sentences, than high semantic predictability. The model also computed a significant interaction between SNR and Semantic Predictability: low predictability sentences at a low SNR (-6 dB SNR) had even lower keyword identification accuracy.

This interaction is depicted in Figure 1: in high semantic predictability contexts and a low SNR, i.e., the most optimal listening condition, keyword identification accuracy is at ceiling and there is no difference between TTS voice types. Yet, as adverse listening increases for these sentences, there is a large difference in accuracy for the TTS types, with greater accuracy for concatenative TTS. In low semantic predictability contexts, listeners are more accurate for concatenative TTS than neural TTS at a lower SNR.

\section{Accuracy in keyword identification}

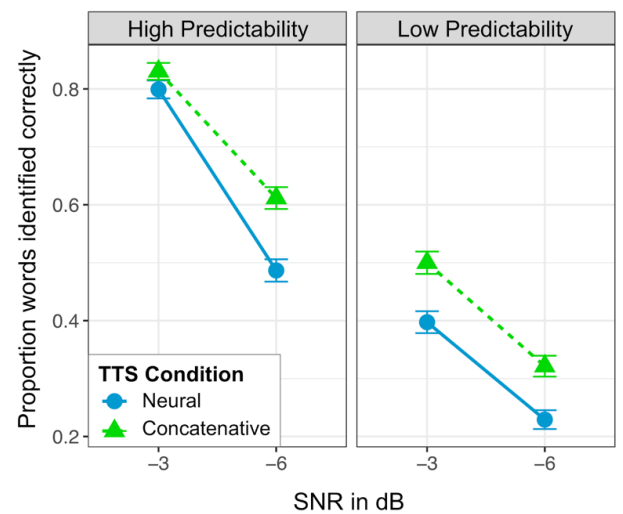

Figure 1. Mean accuracy of keyword identification in high and low Semantic Predictability contexts, lower and higher SNR, across two TTS synthesis types. Error bars $=$ standard error.

Table 1. Model summary for word identification accuracy

\begin{tabular}{|c|c|c|c|c|c|}
\hline & $\begin{array}{l}\text { Beta } \\
\text { Coef }\end{array}$ & $\begin{array}{l}\text { Std } \\
\text { Error }\end{array}$ & $z$ & $p$ & \\
\hline (Intercept) & 0.13 & 0.07 & 1.81 & 0.07 & \\
\hline TTS_Neural & -0.21 & 0.03 & -6.16 & $<0.001$ & $* * *$ \\
\hline SNR_-6 & -0.53 & 0.03 & -16.55 & $<0.001$ & $* * *$ \\
\hline Predict_Low & -0.73 & 0.03 & -23.65 & $<0.001$ & $* * *$ \\
\hline TTS_Neural $*$ SNR_-6 & -0.04 & 0.03 & -1.29 & 0.20 & \\
\hline $\begin{array}{l}\text { TTS_Neural* } \\
\text { Predict_Low }\end{array}$ & -0.02 & 0.03 & -0.53 & 0.59 & \\
\hline SNR_-6 * Predict_Low & 0.013 & 0.03 & 4.33 & $<0.001$ & $* * *$ \\
\hline $\begin{array}{l}\text { TTS_Neural* SNR_-6 } \\
\text { * Predict_Low }\end{array}$ & 0.03 & 0.03 & 0.94 & 0.35 & \\
\hline
\end{tabular}

\section{Experiment 2: Language Attitudes}

\subsection{Methods}

\subsubsection{Participants}

The same participants who participated in Experiment 1 competed Experiment 2 (§ 2.1.1).

\subsubsection{Procedure}

Following Experiment 1, and the hearing screen, participants completed a language attitudes study, where they heard a single sentence ("The girl knows about the swamp.") produced by each of the 4 speakers in the 2 TTS conditions ( 8 voices in total) and provided ratings of the voice using a sliding scale (0-100): (1) How machine-like/human-like, (2) How unfamiliar/familiar?, (3) How eerie/natural? and (4) How unlikeable/likeable? Order of neural and concatenative TTS 
voices was blocked, so that the TTS for the same speaker was not presented sequentially. The ratings task was also blocked by question: Participants provided a rating for each of the 8 voices for a given dimension (e.g., 'human-likeness').

\subsubsection{Analysis}

Participants' ratings (machine-like/human-like, unfamiliar/ familiar, eerie/natural) of the voices were analyzed using separate linear mixed effects models with the lme $4 \mathrm{R}$ package [31]. Fixed effects included TTS Condition, while the random effects included by-Listener and by-Speaker random intercepts.

\subsection{Results}

As seen in Figure 2., all models showed a similar main effect of TTS Condition: listeners rated the neural TTS voices as more human-like $[\beta=9.92, t=8.6, p<0.001]$, likeable $[\beta=8.5$, $t=6.4, p<0.001]$, natural $[\beta=8.5, t=6.3, p<0.001]$, and familiar $[\beta=3.50, t=2.0, p<0.05]$ than the concatenative TTS.

\section{Relating Intelligibility and Ratings}

To investigate whether there was a relationship between an individual participant's rating for a given voice (e.g., 'Salli' neural) and their word identification accuracy for that voice, we conducted a post-hoc analysis. We modeled word identification accuracy in separate mixed effects logistic regression models (for the 4 ratings: familiar, human-like, natural, and likeable), with the fixed effect of TTS Condition, Rating Score (continuous, z-scored within speaker/rating), their interaction, and by-Subject and by-Speaker random intercepts.

All four models showed no main effect of the rating on accuracy. However, two of the models revealed significant interactions: word identification accuracy was higher for the neural TTS voices when they were rated as being more human-like $[\beta=0.09, t=2.9, p<0.01]$ or more natural $[\beta=0.06$, $t=2.0, p<0.05]$. There was no effect of familiarity $[\beta=-0.05$, $t=1.7, p=0.10]$ or likeability $[\beta=0.05, t=1.8, p=0.08]$ by TTS condition.

\section{Discussion}

The present study investigated whether the type of TTS synthesis method, concatenative or neural, results in different listener perception patterns. Experiment 1 compared speechin-noise perception of neural and concatenative TTS generated from identical speaker datasets. Experiment 2 compared listeners' language attitudes of concatenative and neural TTS, again generated from the same set of speakers.

In Experiment 1, we found, overall, that neural TTS, resulted in reduced speech-in-noise perception, relative to the concatenative TTS method. This result is in line with prior research indicating that more casual, connected speech results in less information for listeners to glean [13]. This finding is relevant both for models of speech perception - in the role of 'clear' versus 'connected' speech in speech-in-noise perception - as well as for applications to speech technology. For one, this finding suggests that neural TTS, while increasingly naturalistic, may actually reduce listeners' ability to perceive what a modern voice-AI system may be communicating, if it's being used in the presence of competing noise, such as in a noisy room or multiple background talkers. Additionally, we found that listeners integrate semantic

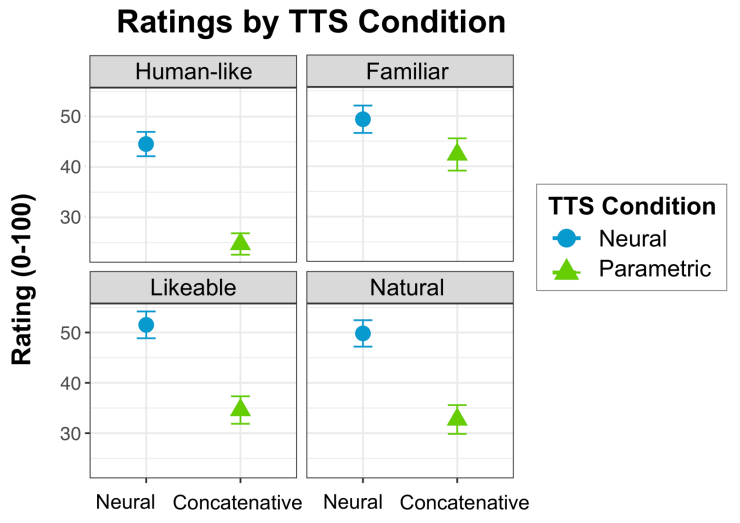

Figure 2. Mean ratings for TTS type: neural (blue circle) vs. concatenative (green triangle). Error bars = standard error.

context differently for TTS voices varying in human-likeness. In low predictability contexts, neural TTS is even less intelligible than concatenative TTS with increasing listening difficulty.

Meanwhile, in Experiment 2, we observed differences in listeners' ratings of the concatenative vs. neural TTS voices for four social attributes: listeners rate neural TTS voices as more human-like, natural, and familiar, and more likeable than concatenative TTS voices, consistent with prior work (e.g., [2]).

Finally, we linked the results from Experiments $1 \& 2$ : we found evidence of individual variation mediating intelligibility in the word identification task. A given listener's ratings of how human-like or natural they found the voices correlated with their overall performance accuracy for identifying words in the neural TTS voices: voices that were rated as sounding more natural and more human-like showed less of a decrease in intelligibility than voices that were rated as less natural and less human-like. One possibility is that a listener who rated the voice as sounding less human-like may assume they would not be able to understand the TTS; this would be in line with findings of accented speech, where a listener's attitudes of that variety shapes their ability to perceive that individual across listening conditions (e.g., [24]). Another possibility is that the effect is driven by attention: we hypothesize that listeners might pay closer attention to the acoustic-phonetic details for voices they perceive as sounding more human-like.

Yet, several questions still remain to be addressed, which can serve as areas for future research. For one, which acousticphonetic features demonstrate stronger coarticulation in neural TTS has not been explored. An in-depth investigation of multiple coarticulatory patterns would be insightful to quantify why neural TTS voices sound more human-like.

Overall, this study has implications for voice user interface design. For one, it is noteworthy that the more advanced and realistic TTS method results in less intelligible speech in adverse listening conditions. Further work exploring this effect across different types of background noise (e.g., 1talker, multitalker babble) and across listeners (e.g., older individuals, hearing-impaired individuals, individuals with autism) can be insightful for tailoring TTS. Additionally, this result suggests having a user choose the voice that sounds most human-like and natural to them may aid intelligibility across listening conditions, even if this speech synthesis method is less intelligible in adverse listening conditions overall. 


\section{References}

[1] M. Cooke, C. Mayo, and C. Valentini-Botinhao, "Intelligibilityenhancing speech modifications: the hurricane challenge.," in Interspeech, 2013, pp. 3552-3556.

[2] A. Van Den Oord et al., "WaveNet: A generative model for raw audio.," in $S S W, 2016$, p. 125.

[3] Z. Malisz, G. E. Henter, C. Valentini-Botinhao, O. Watts, J. Beskow, and J. Gustafson, "Modern speech synthesis for phonetic sciences: A discussion and an evaluation," in Proceedings of ICPhS, 2019.

[4] T. Merritt et al., "Comprehensive Evaluation of Statistical Speech Waveform Synthesis," in 2018 IEEE Spoken Language Technology Workshop (SLT), Dec. 2018, pp. 325-331, doi 10.1109/SLT.2018.8639556.

[5] S. Ronanki, "Prosody generation for text-to-speech synthesis," Dissertation, University of Edinburgh, 2019

[6] E. Farnetani and D. Recasens, "Coarticulation and connected speech processes,” Handb. Phon. Sci., pp. 371-404, 1997.

[7] P. Assmann and Q. Summerfield, "The perception of speech under adverse conditions," in Speech processing in the auditory system, Springer, 2004, pp. 231-308.

[8] M. Fallon, S. E. Trehub, and B. A. Schneider, "Children's perception of speech in multitalker babble," J. Acoust. Soc. Am., vol. 108, no. 6, pp. 3023-3029, 2000.

[9] M. N. Ruscetta, E. M. Arjmand, and S. R. Pratt, "Speech recognition abilities in noise for children with severe-toprofound unilateral hearing impairment," Int. J. Pediatr. Otorhinolaryngol., vol. 69, no. 6, pp. 771-779, 2005.

[10] P. E. Souza and C. W. Turner, "Masking of speech in young and elderly listeners with hearing loss," J. Speech Lang. Hear. Res., vol. 37, no. 3, pp. 655-661, 1994.

[11] M. L. G. Lecumberri and M. Cooke, "Effect of masker type on native and non-native consonant perception in noise," J. Acoust. Soc. Am., vol. 119, no. 4, pp. 2445-2454, Mar. 2006, doi: 10.1121/1.2180210.

[12] O. Simantiraki, M. Cooke, and S. King, "Impact of Different Speech Types on Listening Effort.," in Interspeech, 2018, pp. 2267-2271

[13] L. Shockey, "Phonetic and phonological properties of connected speech," The Ohio State University, 1973.

[14] R. A. Scarborough, "Lexical confusability and degree of coarticulation," in Annual Meeting of the Berkeley Linguistics Society, 2003, vol. 29, pp. 367-378.

[15] I. V. Bele, “The Speaker's Formant," J. Voice, vol. 20, no. 4, pp. 555-578, Dec. 2006, doi: 10.1016/j.jvoice.2005.07.001.

[16] C. G. Clopper, "Effects of dialect variation on the semantic predictability benefit," Lang. Cogn. Process., vol. 27, no. 7-8, pp. 1002-1020, Sep. 2012, doi: 10.1080/01690965.2011.558779.

[17] S. Kennedy and P. Trofimovich, "Intelligibility,

Comprehensibility, and Accentedness of L2 Speech: The Role of Listener Experience and Semantic Context," Can. Mod. Lang. Rev., Mar. 2008, doi: 10.3138/cmlr.64.3.459.

[18] S. V. van der Feest, C. P. Blanco, and R. Smiljanic, "Influence of speaking style adaptations and semantic context on the time course of word recognition in quiet and in noise," J. Phon., vol. 73, pp. 158-177, 2019.

[19] K. L. Payton, R. M. Uchanski, and L. D. Braida, "Intelligibility of conversational and clear speech in noise and reverberation for listeners with normal and impaired hearing," J. Acoust. Soc. Am. vol. 95 , no. 3, pp. 1581-1592, 1994

[20] M. A. Picheny, N. I. Durlach, and L. D. Braida, "Speaking clearly for the hard of hearing II: Acoustic characteristics of clear and conversational speech," J. Speech Lang. Hear. Res., vol. 29, no. 4, pp. 434-446, 1986.

[21] L. E. Humes, B. U. Watson, L. A. Christensen, C. G. Cokely, D. C. Halling, and L. Lee, "Factors associated with individual differences in clinical measures of speech recognition among the elderly," J. Speech Lang. Hear. Res., vol. 37, no. 2, pp. 465-474, 1994

[22] J. I. Alcántara, E. J. Weisblatt, B. C. Moore, and P. F. Bolton, "Speech-in-noise perception in high-functioning individuals with autism or Asperger's syndrome," J. Child Psychol. Psychiatry, vol. 45, no. 6, pp. 1107-1114, 2004.

[23] K. J. Van Engen, "Similarity and familiarity: Second language sentence recognition in first- and second-language multi-talker babble," Speech Commun., vol. 52, no. 11, pp. 943-953, Nov. 2010, doi: 10.1016/j.specom.2010.05.002.

[24] M. Babel and J. Russell, "Expectations and speech intelligibility," J. Acoust. Soc. Am., vol. 137, no. 5, pp. $2823-$ 2833, 2015.

[25] S. V. Berg, A. Panorska, D. Uken, and F. Qeadan, "DECtalk ${ }^{\mathrm{TM}}$ and VeriVox ${ }^{\mathrm{TM}}$ : Intelligibility, Likeability, and Rate Preference Differences for Four Listener Groups," Augment. Altern. Commun., vol. 25, no. 1, pp. 7-18, Jan. 2009, doi: $10.1080 / 07434610902728531$.

[26] D. N. Kalikow, K. N. Stevens, and L. L. Elliott, "Development of a test of speech intelligibility in noise using sentence materials with controlled word predictability," J. Acoust. Soc. Am., vol. 61, no. 5, pp. 1337-1351, 1977.

[27] H. Quené and L. E. Van Delft, "Non-native durational patterns decrease speech intelligibility," Speech Commun., vol. 52, no. 11-12,pp. 911-918, 2010.

[28] M. Winn, Make speech-shaped noise. 2019.

[29] D. McCloy, Mix speech with noise. 2015.

[30] J. Reilly, V. Troiani, M. Grossman, and R. Wingfield, "An introduction to hearing loss and screening procedures for behavioral research," Behav. Res. Methods, vol. 39, no. 3, pp. 667-672, Aug. 2007, doi: 10.3758/BF03193038.

[31] D. Bates, M. Mächler, B. Bolker, and S. Walker, "Fitting Linear Mixed-Effects Models Using lme4," J. Stat. Softw., vol. 67, no. 1, pp. 1-48, Oct. 2015, doi: 10.18637/jss.v067.i01. 